Mona.tshefte für Chemie 107, 1271-1279 (1976)

O by Springer-Verlag 1976

\title{
Isoelektronische Derivate des Sila-Clofenetamins und des Sila-Mebrophenhydramins
}

\author{
(Sila-Pharmaka, 6. Mitt. ${ }^{\text {I }}$ ) \\ Von \\ Reinhold Tacke ${ }^{2}$ und Ürich Wannagat \\ Institut für 'Anorganische Chemie, Technische Universität Braunschweig, \\ Bundesrepublik Deutschland
}

(Eingegangen am 10. Mai 1976)

\begin{abstract}
Isoelectronic Derivatives of Sila-Clofenetamine and Sila-Mebrophenhydramine
\end{abstract}

Isoelectronic derivatives (A and B) and a homolog (G) of the two sila-antihistamines sila-clofenetamine and silamebrophenhydramine were synthesized for the first time by the steps shown in scheme 1. They and their unknown precursors II-IV were characterized by their physical (Table 1) and chemical properties and their structures confirmed by ${ }^{1} \mathrm{H}-\mathrm{NMR}$ and mass spectroscopy (Tables 2 and 3 ). The pharmacological effects of $\mathbf{A}$ and $\mathbf{B}$ were investigated and compared with those of the corresponding $\mathrm{O}$-isosteric sila-antihistamines (Chapter 5).

\section{Einführung}

Im Rahmen unserer Arbeiten über Sila-Pharmaka konnten wir bereits früher verschiedene Silicium-Analoga der BenzhydrylätherAntihistaminika $^{3-5}$ synthetisieren. Die bisher untersuchten Vertreter dieser Verbindungs-Klasse $\left[\mathrm{X}=\mathrm{CH}_{3}, \mathrm{H}, \mathrm{Cl}, \mathrm{Br}\right.$ und $\mathrm{NR}_{2}=\mathrm{N}\left(\mathrm{CH}_{3}\right)_{2}$, $\mathrm{N}\left(\mathrm{C}_{2} \mathrm{H}_{5}\right)_{2}$, Morpholino] besitzen $u$. a. histaminolytische, anticholinerge

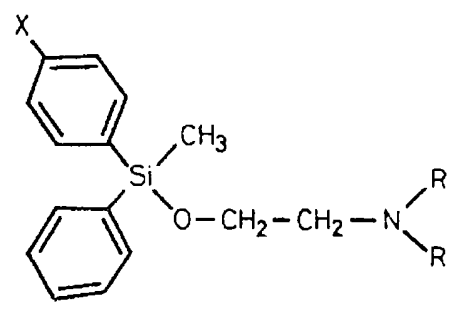

und muskulotrop spasmolytische Eigenschaften ${ }^{6}$, wobei die erstgenannte Wirkqualität stets am stärksten ausgeprägt ist. 
Während die pharmakologischen Wirkungen durch Variation von $X$ und $\mathrm{R}$ nur wenig beeinflußt werden, führen andere Strukturabwandlungen $\mathrm{zu}$ starken Veränderungen. So bedingt sowohl eine $\mathrm{N}$ Quaternierung ${ }^{7}$ als auch ein Austausch einer Phenylgruppe gegen eine Norbornenylgruppe ${ }^{8}$ einen deutlichen Verlust an histaminolytischer Aktivität und einen starken Gewinn anticholinerger Wirkung. Die Verlängerung der Aminoalkoxygruppe ${ }^{1}$ durch eine $\mathrm{CH}_{2}-\mathrm{Gruppe}$ sowie die Substitution ${ }^{1}$ einer $\mathrm{Si}-\mathrm{CH}_{3}$ - durch eine $\mathrm{Si}-\mathrm{C}_{2} \mathrm{H}_{5}$-Gruppe führt ebenfalls zu einer erheblichen Abschwächung der histaminolytischen Aktivität, während die beiden anderen Wirkqualitäten hierdurch nur wenig verändert werden.

Allen bisher dargestellten Sila-benzhydryläthern und ihren Derivaten ist eine kurze Wirkungsdauer ( $<30$ Min.) gemeinsam, da die Verbindungen an der Si-O-C-Bindung hydrolytisch gespalten werden.

Skizze 1. Zur Darstellung der isoelektronischen Derivate A und B des Sila-Clofenetamins und des Sila-Mebrophenhydramins

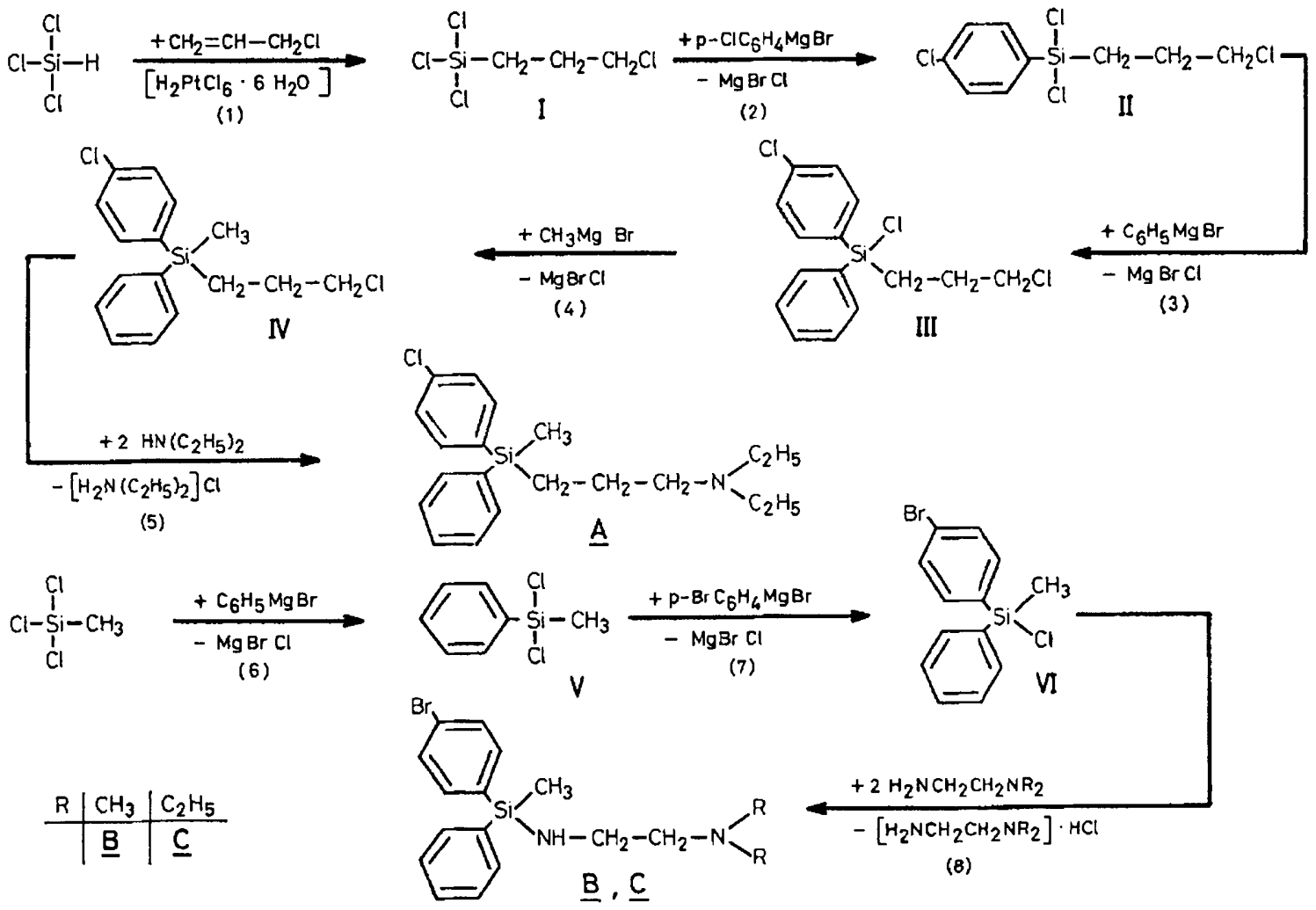

In Fortführung unserer Untersuchungen an Sila-benzhydryläthern haben wir weitere Veränderungen am Strukturgerüst dieser Verbindungen vorgenommen und in Hinblick auf die biologische Aktivität und das Hydrolyseverhalten untersucht. Im folgenden soll über die 
Darstellung und Eigenschaften von isoelektronischen Derivaten des Sila-Clofenetamins $\left(X=\mathrm{Cl}, \mathrm{R}=\mathrm{C}_{2} \mathrm{H}_{5}\right)$ und Sila-Mebrophenhydramins $\left(X=\mathrm{Br}, \mathrm{R}=\mathrm{CH}_{3}\right.$ ) berichtet werden, bei denen das O-Atom durch eine $\mathrm{CH}_{2}$-Gruppe $(\rightarrow \mathrm{A})$ bzw. eine NH-Gruppe $(\rightarrow \mathrm{B})$ ersetzt ist.

2. Die Darstellung der isosteren Sila-benzhydrylätherDerivate A, B und C sowie ihrer Vorstufen

Zur Darstellung von A wurde zunächst nach bekanntem Verfahren ${ }^{9}$ durch Addition (Rk. 1) von Allylchlorid an Trichlorsilan (in Gegenwart katalytischer Mengen $\mathrm{H}_{2} \mathrm{PtCl}_{6} \cdot 6 \mathrm{H}_{2} \mathrm{O}$ ) 3-Chlorpropyl-trichlorsilan (I) synthetisiert. Dieses ließ sich durch Umsetzung mit p-Chlorphenylmagnesiumbromid (Rk. 2) in II und durch weitere Reaktion mit Phenylmagnesiumbromid (Rk. 3) in III überführen. Durch eine letzte Grignardstufe mit Methylmagnesiumbromid (Rk.4) entstand IV, das durch Umsetzung mit Diäthylamin im Bombenrohr (Rk.5) schließlich A ergab (s. Skizze 1).

Zur Darstellung von. B und $\mathbf{C}$ wurde, von Methyltrichlorsilan ausgehend, nach bekanntem Verfahren durch stufenweise Reaktion mit Phenylmagnesiumbromid (Rk. 6) ${ }^{10}$ und p-Bromphenylmagnesiumbromid (Rk. 7) ${ }^{11}$ über $V$ zunächst VI synthetisiert. Dieses reagierte gemäß Rk. (8) mit den entsprechenden N,N-Dialkyläthylendiaminen zu B bzw. C (s. Skizze 1).

\section{Experimenteller Teil}

Die Darstellung von I, V und VI erfolgte in Anlehnung an die angegebenen Literaturzitate.

Die Synthese von II-IV gelang in der üblichen Weise durch Grignardreaktionen, die problemlos abliefen; bei der Aufarbeitung traten aber Schwierigkeiten auf, da sich die Verbindungen bei der Destillation aus den Rohprodukten z. T. durch $\gamma$-Eliminierung zersetzten (besonders III und IV). So wurde z. B. bei der Vakuumdestillation von III aus dem Rohprodukt mehrfach eine fast quantit. Zersetzung zu p-Chlorphenyl-phenyldichlorsilan beobachtet. Durch eine möglichst vollständige Entfernung der Mg-Salze und von nicht umgesetztem Grignardreagens (durch Fällung mit $T H F$ und $P \ddot{A}$ und nachfolgende Filtration über wasserfr. $\mathrm{Na}_{2} \mathrm{SO}_{4}$ ) konnte die $\gamma$-Eliminierung jedoch weitgehend unterdrückt werden. Zur Isolierung von reinem IV erwies es sich außerdem als nützlich, vor der Destillation etwas Diäthylamin zuzugeben.

\section{3-Chlorpropyl-(p-chlorphenyl)-dichlorsilan (II)}

Gemäß Rk. (2) tropft man zu $212 \mathrm{~g}$ (1 Mol) I in $500 \mathrm{ml}$ Diäthyläther $(=\ddot{A})$ eine Lösung von $1 \mathrm{Mol} \mathrm{p}$-Chlorphenylmagnesiumbromid in $800 \mathrm{ml}$ $\dddot{A}$, rührt 2 Stdn. unter Rückfluß weiter, tropft anschließend in der Hitze langsam $200 \mathrm{ml} T H F$ hinzu, rührt $12 \mathrm{Stdn}$. bei $20^{\circ} \mathrm{C}$, filtriert von den Mg-Salzen, zieht das Lösungsmittel bei $20^{\circ} \mathrm{C}$ im Vak. möglichst vollständig 
ab, nimmt den Rückstand in $600 \mathrm{ml}$ Petroläther $\left(40 / 65^{\circ} \mathrm{C}=P \ddot{A}\right)$ auf, filtriert über eine Schicht von wasserfr. $\mathrm{Na}_{2} \mathrm{SO}_{4}$ und zieht das Lösungsmittel bei $20^{\circ} \mathrm{C}$ im Vak. vollständig ab. Anschließend nimmt man den Rückstand erneut in $600 \mathrm{ml} P \ddot{A}$ auf und filtriert nochmals über $\mathrm{Na}_{2} \mathrm{SO}_{4}$, zieht das Lösungsmittel ab und destilliert im Vak. über eine Vigreux-, anschließend über eine Drehbandkolonne. Ausb. 132,5 g II (46\%).

\section{3-Chlorpropyl-(p-chlorphenyl)-phenyl-chlorsilan (III)}

Gemäß Rk. (3) durch Zutropfen einer Lösung von $0,2 \mathrm{Mol} \mathrm{C}_{6} \mathrm{H}_{5} \mathrm{MgBr}$ in $160 \mathrm{ml} \ddot{A}$ zu $57,6 \mathrm{~g}(0,2 \mathrm{Mol})$ II in $100 \mathrm{ml} \ddot{A}$. Reaktionsbedingungen und Aufarbeitung analog zu Rk. (2). Ausb, 26,4 g III (40\%).

\section{3-Chlorpropyl-(p-chlorphenyl)-methyl-phenylsilan (IV)}

Gemäß Rk. (4) durch Zutropfen einer Lösung von $0,1 \mathrm{Mol} \mathrm{CH}_{3} \mathrm{MgBr}$ in $200 \mathrm{ml} \ddot{A}$ zu $33 \mathrm{~g}(0,1 \mathrm{Mol})$ III in $50 \mathrm{ml} \ddot{A}$. Anschließend 2 Stdn. Rückfluß, Fällung von Mg-Salzen mit $50 \mathrm{ml} \mathrm{THF}, 12 \mathrm{Stdn}$. Rühren bei $20^{\circ} \mathrm{C}$, Filtration von den Mg-Salzen, Abziehen des Lösungsmittels bei $20^{\circ} \mathrm{C}$ im Vak., Aufnehmen des Rückstandes in $250 \mathrm{ml} P \ddot{A}$ und Filtration über $\mathrm{Na}_{2} \mathrm{SO}_{4}$. Das Filtrat wird anschließend mit 4,4 $\mathrm{g}$ Diäthylamin versetzt. Man läßt 30 Min. stehen, filtriert von den ausgefallenen Salzen über $\mathrm{Na}_{2} \mathrm{SO}_{4}$, zieht das Lösungsmittel ab und destilliert den Rückstand im Vak. zunächst über eine Vigreux-, dann über eine Drehbandkolonne. Ausb. 16,4 g IV $(53 \%)$.

N,N-Diäthyl-3-(p-chlorphenyl-methyl-phenylsilyl)-propylamin (A) Rk. (5)

Man bringt $15,5 \mathrm{~g}(0,05 \mathrm{Mol}) \mathrm{IV}$ und $18,3 \mathrm{~g}(0,25 \mathrm{Mol})$ Diäthylamin für $15 \mathrm{Stdn}$. im Bombenrohr bei $150^{\circ} \mathrm{C}$ zur Reaktion, versetzt das Reaktionsgemisch anschließend mit $150 \mathrm{ml} P \ddot{A}$, filtriert vom ausgefallenen $E t_{2} \mathrm{NH} \cdot \mathrm{HCl}$ über eine Schicht von $\mathrm{Na}_{2} \mathrm{SO}_{4}$, zieht das Lösungsmittel ab und fraktioniert im Vak. 2mal über eine Vigreux-Kolonne. In der Hauptfraktion finden sich $14,4 \mathrm{~g}$ A $(83 \%)$.

\section{$N, N$-Dimethyl-N'-(p-bromphenyl-methyl-phenylsilyl)-äthylendiamin (B)}

Rk. (8)

Man tropft zu $17,6 \mathrm{~g}(0,2 \mathrm{Mol}) \mathrm{N}, \mathrm{N}$-Dimethyläthylendiamin in $200 \mathrm{ml}$ $P \ddot{A}$ bei $20^{\circ} \mathrm{C}$ langsam und unter starkem Rühren eine Lösung von $15,6 \mathrm{~g}$ $(0,05 \mathrm{Mol})$ VI in $100 \mathrm{ml} P \ddot{A}$, wobei sofort das Hydrochlorid des Diamins ausfällt. Nach Beendigung des Zutropfens rührt man $1 \mathrm{Stde}$. bei $20^{\circ} \mathrm{C}$, dann 4 Stdn. unter Rückfluß weiter, filtriert vom Hydrochlorid, wäscht den Niederschlag mit jeweils $50 \mathrm{ml} P \ddot{A}$, zieht das Lösungsmittel ab, destilliert den Rückstand im Vak., nimmt die Hauptfraktion anschließend in $150 \mathrm{ml}$ $P \ddot{A}$ auf, filtriert über eine Schicht von $\mathrm{Na}_{2} \mathrm{SO}_{4}$ von erneut ausgefallenem Hydrochlorid, zieht das Lösungsmittel ab und fraktioniert im Vak. 2mal über eine Vigreux-Kolonne. Ausb. $12,9 \mathrm{~g}$ B (71\%).

N,N-Diäthyl-N'-(p-bromphenyl-methyl-phenylsilyl)-äthylendiamin (G) Rk. (8)

Analog zur Darstellung von $\mathrm{B}$ aus $23,2 \mathrm{~g} \mathrm{~N}, \mathrm{~N}$-Diäthyl-äthylendiamin und $15,6 \mathrm{~g}$ VI. Ausb. 14,3 g C (73\%). 
3. Chemische und physikalische Eigenschaften der neu dargestellten Verbindungen

Die Vorstufen I, V und VI waren bereits gut bekannt (Darstellung und Eigenschaften: $\left.I^{9}, V^{10},{ }^{12},{ }^{13}, V I^{11}\right)$. Die von uns ermittelten Siedepunkte, Brechungsindices und Dichten stimmten weitgehend mit den Literaturangaben überein ${ }^{2}, 3,5$.

Die neu dargestellten Vorstufen II-IV sind wasserklare, farblose Flüssigkeiten, die sich gut in den üblichen inerten organischen Solventien lösen. Sie sind mit Ausnahme von IV hydrolyseempfindlich.

II-IV wurden bisher noch nicht in der Literatur beschrieben; es wird lediglich über ein Gemisch stellungsisomerer Verbindungen des Typs $M e P h\left(\mathrm{C}_{6} \mathrm{H}_{4} \mathrm{Cl}\right) \mathrm{Si}\left(\mathrm{CH}_{2}\right)_{3} \mathrm{Cl}$ berichtet ${ }^{14}$, das durch Umsetzung eines entsprechenden Isomerengemisches von $M e P h\left(\mathrm{C}_{6} \mathrm{H}_{4} \mathrm{Cl}\right) \mathrm{SiH}$ mit Allylchlorid erhalten wurde.

A, $\mathbf{B}$ und $\mathbf{C}$ sind ölige Flüssigkeiten, die sich gut in den gängigen organischen Lösungsmitteln wie $P \ddot{A}, E t_{2} \mathrm{O}, \mathrm{C}_{6} \mathrm{H}_{6}, \mathrm{CHCl}_{3}, \mathrm{CH}_{2} \mathrm{Cl}_{2}$ oder $\mathrm{CCl}_{4}$, dagegen schlecht in Wasser lösen. $\mathrm{A}$ ist bei einer Konzentration von $10^{-6} \mathrm{Mol} / \mathrm{l}$ in Tyrodelösung $\left(\mathrm{pH} 7,4,37^{\circ} \mathrm{C}\right.$ ) über mehr als 2 Stdn, stabil, während $\mathbf{B}$ und $\mathbf{C}$ unter den gleichen Bedingungen durch hydrolytische Spaltung der $\mathrm{Si}-\mathrm{N}$-Bindung offensichtlich so rasch zerfallen, daß im Gegensatz zu A und den O-Isosteren keine pharmakologischen Effekte der $\mathrm{Si}-\mathrm{N}$-Verbindungen gemessen werden konnten (s. Kap. 5).

A, B und $\mathbf{C}$ sind im Vak. unzersetzt destillierbar. Bemerkenswert ist, daß bei der Destillation der 3-Chlorpropylsilane II-IV aus den Rohprodukten z. T. eine $\gamma$-Eliminierung auftritt. Diese ist vermutlich auf die Anwesenheit von Mg-Halogeniden und (oder) nicht umgesetztem Grignard-Reagens zurückzuführen. Die reinen Silane II-IV sind dagegen weitgehend unzersetzt im Vak. destillierbar.

\section{Zur Strukturermittlung der neu dargestellten Verbindungen}

wurden neben den Elementaranalysen (Tab. 1) vor allem 1H.NMR. und Massenspektren herangezogen.

Die Kernresonanzspektren (Tab. 2) sind mit ihren vielen Signalgruppen auf Grund der charakteristischen chemischen Verschiebung und Multiplizität sehr nützlich und aussagekräftig. Die gemessenen Daten liegen weitgehend alle im Erwartungsbereich.

Die Massenspektren (Tab. 3) erwiesen sich auf Grund der charakteristischen Halogenisotopenmuster ebenfalls als sehr aussagekräftig. $\mathbf{B}$ und $\mathbf{G}$ zeigen in ihrem Fragmentierungsverhalten eine gewisse Verwandtschaft mit den O-isosteren Verbindungen ${ }^{3-5}$. Bemerkenswert 
ist allerdings, daß in den Spektren von $\mathbf{B}$ und $\mathbf{C}$ neben den aus einer $\alpha$-Spaltung hervorgegangenen $\mathrm{CH}_{2}=\mathrm{NR}_{2}{ }^{\oplus}$-Ionen auch die entsprechenden $خ \mathrm{Si}-\mathrm{NH}=\mathrm{CH}_{2}{ }^{\oplus}$-Ionen auftreten. In den Spektren (RoutineMessungen) der $\mathrm{O}$-Isosteren sind nur die $\mathrm{CH}_{2}=\mathrm{NR}_{2}{ }^{\oplus}$-Ionen auffindbar. Sie liefern in jedem Fall den Basispeak. Im Massenspektrum von A dominiert ebenfalls das durch $\alpha$-Spaltung entstehende $\mathrm{CH}_{2}=\mathrm{N}\left(\mathrm{C}_{2} \mathrm{H}_{5}\right)_{2}{ }^{\oplus}$-Ion.

Tabelle 1. Physikalische und elementaranalytische ${ }^{\text {a }}$ Daten der neu dargestellten Verbindungen

\begin{tabular}{|c|c|c|c|c|c|c|}
\hline $\begin{array}{l}\text { Lfd. } \\
\mathrm{Nr} .\end{array}$ & Summenformel b & $\begin{array}{c}\text { Sdp., } \\
\text { 'C/Torr }\end{array}$ & $D_{4}^{20}$ & $n_{\mathrm{D}}^{20}$ & $\begin{array}{c}\% \mathrm{C} \\
\text { gef. } \\
\text { (ber.) }\end{array}$ & $\begin{array}{c}\% \mathrm{H} \\
\text { gef. } \\
\text { (ber.) }\end{array}$ \\
\hline II & $\mathrm{C}_{9} \mathrm{H}_{10} \mathrm{Cl}_{4} \mathrm{Si}$ & $119-120 / 1$ & 1,3364 & 1,5489 & $\begin{array}{c}37,68 \\
(37,52)\end{array}$ & $\begin{array}{c}3,59 \\
(3,50)\end{array}$ \\
\hline III & $\mathrm{C}_{15} \mathrm{H}_{15} \mathrm{Cl}_{3} \mathrm{Si}$ & $155-157 / 0,01$ & 1,2448 & 1,5851 & $\begin{array}{c}53,84 \\
(54,64)\end{array}$ & $\begin{array}{r}4,46 \\
(4,59)\end{array}$ \\
\hline IV & $\mathrm{C}_{16} \mathrm{H}_{18} \mathrm{Cl}_{2} \mathrm{Si}$ & $155-157 / 0,2$ & 1,1457 & 1,5773 & $\begin{array}{c}61,65 \\
(62,13)\end{array}$ & $\begin{array}{r}5,75 \\
(5,87)\end{array}$ \\
\hline $\mathbf{A}$ & $\mathrm{C}_{20} \mathrm{H}_{28} \mathrm{ClNSi}$ & $179-181 / 0,5$ & 1,0269 & 1,5490 & $\begin{array}{c}69,50 \\
(69,43)\end{array}$ & $\begin{array}{c}8,04 \\
(8,16)\end{array}$ \\
\hline $\mathbf{B}$ & $\mathrm{C}_{17} \mathrm{H}_{23} \mathrm{BrN}_{2} \mathrm{Si}$ & $154-155 / 0,1$ & 1,2000 & 1,5693 & $\begin{array}{c}55,07 \\
(56,19)\end{array}$ & $\begin{array}{c}6,27 \\
(6,38)\end{array}$ \\
\hline $\mathbf{C}$ & $\mathrm{C}_{19} \mathrm{H}_{27} \mathrm{BrN}_{2} \mathrm{Si}$ & $183-184 / 0,5$ & 1,1663 & 1,5585 & $\begin{array}{c}57,75 \\
(58,30)\end{array}$ & $\begin{array}{c}6,86 \\
(6,95)\end{array}$ \\
\hline
\end{tabular}

a Weitere elementaranalytische Daten sind in ${ }^{2}$ aufgeführt.

b Molgew. bestätigt durch Molpeak im Massenspektrum (Tab. 3).

Bemerkenswert ist, daß die Verbindungen II-IV im Massenspektrometer einer $\mathrm{C}_{3} \mathrm{H}_{6}$-Eliminierung (Cyclopropan oder Propylen?) unterliegen. Im Massenspektrum von I ergibt das $\mathrm{C}_{3} \mathrm{H}_{6}{ }^{\oplus} \cdot$-Ion sogar den intensivsten Peak und ist auch im Spektrum von II noch mit einer Intensität von $44 \%$ vorhanden. Dieses besondere Verhalten dürfte auf die gemeinsame 3-Chlorpropylgruppe zurückzuführen sein. In den Spektren von I-IV (Routine-Messungen) existieren keine Über. gangssignale für eine elektronenstoßinduzierte $\mathrm{C}_{3} \mathrm{H}_{6}$-Eliminierung, so daß wir nicht mit Sicherheit sagen können, ob dieser Prozeß thermisch oder elektronenstoßinduziert ist.

\section{Pharmakologische Eigenschaften von A und B}

Die pharmakologischen Untersuchungen wurden in enger Zusammenarbeit am Institut für Pharmakologie und Toxikologie der Technischen Universität Braunschweig von H.-U. Rossée und F. Meyer durch- 
Tabelle 2. ${ }^{1} \mathrm{H}$-NMR-Spektren der neu dargestellten Verbindungen, Chemische Verschiebungen in $\delta[\mathrm{ppm}]$, Lösungsmittel: $\mathrm{CCl}_{4}$ (II-IV, A) bzw. $\mathrm{CDCl}_{3}$ (B und $\mathbf{C})$, Standard intern: TMS

\begin{tabular}{|c|c|c|c|c|}
\hline $\begin{array}{l}\text { Lfd. } \\
\text { Nr. }\end{array}$ & $\delta^{1} \mathrm{H}$ & Multiplizität & rel. Intensität & Zuordnung \\
\hline II & $\begin{array}{l}1,0-2,4 \\
3,48 \\
7,48 \mathrm{a}\end{array}$ & $\begin{array}{l}\mathrm{m} \\
\mathrm{t} \\
\mathrm{m}\end{array}$ & $\begin{array}{l}4 \\
2 \\
4\end{array}$ & $\begin{array}{l}\mathrm{SiCH}_{2} \mathrm{CH}_{2} \mathrm{C} \\
\mathrm{CCH}_{2} \mathrm{Cl} \\
\mathrm{SiC}_{6} \mathrm{H}_{4} \mathrm{Cl}\end{array}$ \\
\hline III & $\begin{array}{l}1,0-2,3 \\
3,42 \\
7,0-7,8\end{array}$ & $\begin{array}{l}\mathrm{m} \\
\mathrm{t} \\
\mathrm{m}\end{array}$ & $\begin{array}{l}4 \\
2 \\
9\end{array}$ & $\begin{array}{l}\mathrm{SiCH}_{2} \mathrm{CH}_{2} \mathrm{C} \\
\mathrm{CCH}_{2} \mathrm{Cl} \\
\mathrm{SiC}_{6} \mathrm{H}_{5}, \mathrm{SiC}_{6} \mathrm{H}_{4} \mathrm{Cl}\end{array}$ \\
\hline IV & $\begin{array}{l}0,50 \\
0,7-2,1 \\
3,52 \\
7,0-7,8\end{array}$ & $\begin{array}{l}\mathrm{s} \\
\mathrm{m} \\
\mathrm{t} \\
\mathrm{m}\end{array}$ & $\begin{array}{l}3 \\
4 \\
2 \\
9\end{array}$ & $\begin{array}{l}\mathrm{SiCH}_{3} \\
\mathrm{SiCH}_{2} \mathrm{CH}_{2} \mathrm{C} \\
\mathrm{CCH}_{2} \mathrm{Cl} \\
\mathrm{SiC}_{6} \mathrm{H}_{5}, \mathrm{SiC}_{6} \mathrm{H}_{4} \mathrm{Cl}\end{array}$ \\
\hline $\mathbf{A}$ & $\begin{array}{l}0,50 \\
0,7-1,8 \\
0,94 \\
2,43 \\
2,37 \\
7,0-7,8\end{array}$ & $\begin{array}{l}s \\
m \\
t \\
q \\
t \\
m\end{array}$ & $\left\{\begin{array}{l}3 \\
10 \\
6 \\
9\end{array}\right.$ & $\begin{array}{l}\mathrm{SiCH}_{3} \\
\mathrm{SiCH}_{2} \mathrm{CH}_{2} \mathrm{C} \\
\mathrm{CCH}_{3} \\
\mathrm{NCH}_{2} \mathrm{C} \\
\mathrm{CCCH}_{2} \mathrm{~N} \\
\mathrm{SiC}_{6} \mathrm{H}_{5}, \mathrm{SiC}_{6} \mathrm{H}_{4} \mathrm{Cl}\end{array}$ \\
\hline $\mathbf{B}$ & $\begin{array}{l}0,54 \\
1,5^{\mathrm{b}} \\
1,9-3,1 \\
2,13 \\
7,1-7,8\end{array}$ & $\begin{array}{l}\mathrm{s} \\
\mathrm{m} \\
\mathrm{s} \\
\mathrm{m}\end{array}$ & $\begin{array}{r}3 \\
1 \\
10 \\
9\end{array}$ & $\begin{array}{l}\mathrm{SiCH}_{3} \\
\mathrm{NH} \\
\mathrm{NCH}_{2} \mathrm{CH}_{2} \mathrm{~N} \\
\mathrm{NCH}_{3} \\
\mathrm{SiC}_{6} \mathrm{H}_{5}, \mathrm{SiC}_{6} \mathrm{H}_{4} \mathrm{Br}\end{array}$ \\
\hline $\mathbf{C}$ & $\begin{array}{l}0,56 \\
0,96 \\
1,5^{\mathrm{b}} \\
2,1-3,2 \\
2,47 \\
7,1-7,8\end{array}$ & $\begin{array}{l}\mathrm{s} \\
\mathrm{t} \\
\mathrm{m} \\
\mathrm{q} \\
\mathrm{m}\end{array}$ & $\begin{array}{l}3 \\
6 \\
1 \\
8 \\
9\end{array}$ & $\begin{array}{l}\mathrm{SiCH}_{3} \\
\mathrm{CCH}_{3} \\
\mathrm{NH} \\
\mathrm{NCH}_{2} \mathrm{CH}_{2} \mathrm{~N} \\
\mathrm{NCH}_{2} \mathrm{C} \\
\mathrm{SiC}_{6} \mathrm{H}_{5}, \mathrm{SiC}_{6} \mathrm{H}_{4} \mathrm{Br}\end{array}$ \\
\hline
\end{tabular}

geführt. Für ausführliche Informationen sei auf die Dissertation Rossée verwiesen ${ }^{6}$.

$\mathbf{A}$ und $\mathbf{B}$ wurden am isolierten Meerschweinchen-Ileum in Hinblick auf ihre histaminolytischen, anticholinergen und muskulotrop spasmolytischen Eigenschaften untersucht. Während B überhaupt keine Wirkungen zeigt, sind bei $\mathbf{A}$ alle drei Wirkqualitäten vorhanden. Der Vergleich von A mit dem isosteren Sila-Clofenetamin zeigt jedoch, daß der Ersatz des O-Atoms durch eine $\mathrm{CH}_{2}$-Gruppe mit einem Verlust von etwa drei Vierteln der histaminolytischen Aktivität verbun- 
Tabelle 3. Einige charakteristische Schlüsselbruchstücke aus den Massenspektren der neu dargestellten Verbindungen. Probeneinführung durch

Direkteinlaß, Elektronenstoßionisierung mit $70 \mathrm{eV} .\left(\mathrm{R}=\mathrm{C}_{6} \mathrm{H}_{4} \mathrm{Cl}\right.$, $\left.\mathrm{R}^{\prime}=\mathrm{C}_{6} \mathrm{H}_{4} \mathrm{Br}, M e=\mathrm{CH}_{3}, E t=\mathrm{C}_{2} \mathrm{H}_{5}, P h=\mathrm{C}_{6} \mathrm{H}_{5}\right)$

\begin{tabular}{|c|c|c|c|c|}
\hline $\begin{array}{l}\text { Lfd. } \\
\text { Nr. }\end{array}$ & $m / e$ & $\begin{array}{c}\text { rel. } \\
\text { Intensität }\end{array}$ & $\begin{array}{c}\text { Isotopen- } \\
\text { muster }\end{array}$ & Ion \\
\hline II & $\begin{array}{l}286 / 288 / 290 / 292 / 294 \\
244 / 246 / 248 / 250 / 252 \\
209 / 211 / 213 / 215 \\
174 / 176 / 178 \\
139 / 141 \\
133 / 135 / 137 / 139 \\
63 / 65 \\
42\end{array}$ & $\begin{array}{l}16 / 21 / 10 / 1 /<1 \\
29 / 39 / 19 / 3 /<1 \\
100 / 97 / 34 / 3 \\
96 / 86 / 30 \\
7 / 2 \\
47 / 47 / 15 / 7 \\
45 / 15 \\
44\end{array}$ & $\begin{array}{l}\mathrm{Cl}_{4} \\
\mathrm{Cl}_{4} \\
\mathrm{Cl}_{3} \\
\mathrm{Cl}_{2} \\
\mathrm{Cl} \\
\mathrm{Cl}_{3} \\
\mathrm{Cl}\end{array}$ & $\begin{array}{l}M M^{+} \\
\mathrm{SiRCl}_{3}+ \\
\mathrm{SiRCl}_{2}^{+} \\
\mathrm{SiRCl}+ \\
\mathrm{SiR}^{+} \\
\mathrm{SiCl}_{3}+ \\
\mathrm{SiCl}^{+} \\
\mathrm{C}_{3} \mathrm{H}_{6}+\end{array}$ \\
\hline III & $\begin{array}{l}328 / 330 / 332 / 334 \\
286 / 288 / 290 / 292 \\
251 / 253 / 255 \\
216 / 218 \\
209 / 211 / 213 / 215 \\
105 \\
63 / 65\end{array}$ & $\begin{array}{l}30 / 31 / 11 / 2 \\
64 / 72 / 30 / 3 \\
100 / 94 / 33 \\
33 / 16 \\
25 / 23 / 8 /<1 \\
7 \\
16 / 7\end{array}$ & $\begin{array}{l}\mathrm{Cl}_{3} \\
\mathrm{Cl}_{3} \\
\mathrm{Cl}_{2} \\
\mathrm{Cl} \\
\mathrm{Cl}_{3} \\
\\
\mathrm{Cl}\end{array}$ & $\begin{array}{l}M^{+} \\
\mathrm{SiRPhCl}_{2}+ \\
\mathrm{SiRPh \textrm {Cl } ^ { + }} \\
\mathrm{SiRPh}^{+} \\
\mathrm{SiRCl}_{2}^{+} \\
\mathrm{SiPh}^{+} \\
\mathrm{SiCl}^{+}\end{array}$ \\
\hline IV & $\begin{array}{l}308 / 310 / 312 \\
293 / 295 / 297 \\
266 / 268 / 270 \\
251 / 253 / 255 \\
231 / 233 \\
139 / 141 \\
105 \\
63 / 65\end{array}$ & $\begin{array}{l}<1 /<1 /<1 \\
40 / 27 / 5 \\
23 / 16 / 2 \\
72 / 48 / 8 \\
100 / 33 \\
7 / 3 \\
15 \\
29 / 9\end{array}$ & $\begin{array}{l}\mathrm{Cl}_{2} \\
\mathrm{Cl}_{2} \\
\mathrm{Cl}_{2} \\
\mathrm{Cl}_{2} \\
\mathrm{Cl} \\
\mathrm{Cl}\end{array}$ & $\begin{array}{l}M^{+} \\
M^{+}-M e \\
\text { SiRPhMeCl+ } \\
\text { SiRPhCl+ } \\
\text { SiRPhMe } \\
\mathrm{SiR}^{+} \\
\mathrm{SiPh}^{+} \\
\mathrm{SiCl}^{+}\end{array}$ \\
\hline A & $\begin{array}{l}345 / 347 \\
330 / 332 \\
231 / 233 \\
139 / 141 \\
105 \\
86 \\
58\end{array}$ & $\begin{array}{l}10 / 3 \\
2 /<1 \\
22 / 7 \\
2 /<1 \\
7 \\
100 \\
40\end{array}$ & $\begin{array}{l}\mathrm{Cl} \\
\mathrm{Cl} \\
\mathrm{Cl} \\
\mathrm{Cl}\end{array}$ & $\begin{array}{l}M^{+} \\
M^{+}-M e \\
\mathrm{SiRPhMe}^{+} \\
\mathrm{SiR}^{+} \\
\mathrm{SiPh}^{+} \\
\mathrm{CH}_{2}=\mathrm{N} E t_{2}^{+} \\
\mathrm{CH}_{2}=\mathrm{NH} E t^{+}\end{array}$ \\
\hline B & $\begin{array}{l}362 / 364 \\
347 / 349 \\
304 / 306^{\mathrm{a}} \\
275 / 277^{\mathrm{a}} \\
105 \\
58\end{array}$ & $\begin{array}{l}35 / 37 \\
1 / 1 \\
92 / 95 \\
99 / 100 \\
26 \\
90\end{array}$ & $\begin{array}{l}\mathrm{Br} \\
\mathrm{Br} \\
\mathrm{Br} \\
\mathrm{Br}\end{array}$ & $\begin{array}{l}M^{+} \\
M^{+}-M e \\
\mathrm{CH}_{2}=\mathrm{NH}\left(\mathrm{SiR}^{\prime} P h M e\right)^{+} \\
\mathrm{SiR}^{\prime} P h M e^{+} \\
\mathrm{SiPh}^{+} \\
\mathrm{CH}_{2}=\mathrm{NMe}_{2}{ }^{+}\end{array}$ \\
\hline G & $\begin{array}{l}390 / 392 \\
375 / 377 \\
304 / 306 \\
275 / 277 \\
105 \\
86^{b} \\
58^{b}\end{array}$ & $\begin{array}{l}5 / 6 \\
<1 /<1 \\
5 / 5 \\
22 / 23 \\
8 \\
100 \\
44\end{array}$ & $\begin{array}{l}\mathrm{Br} \\
\mathrm{Br} \\
\mathrm{Br} \\
\mathrm{Br}\end{array}$ & $\begin{array}{l}M^{+} \\
M^{+}-M e \\
\mathrm{CH}_{2}=\mathrm{NH}\left(\mathrm{SiR}^{\prime} P h M e\right)^{+} \\
\mathrm{SiR}^{\prime} P h M e^{+} \\
\mathrm{SiPh}^{+} \\
\mathrm{CH}_{2}=\mathrm{NE} t_{2}{ }^{+} \\
\mathrm{CH}_{2}=\mathrm{NH} E t^{+}\end{array}$ \\
\hline
\end{tabular}

a Der Zerfall von $m / e=304 / 306 \mathrm{zu} m / e=275 / 277$ ist durch ein Übergangssignal bei $m / e=248,7 / 250,8$ belegt.

b Der Zerfall von $m / e=86$ zu $m / e=58$ ist durch das entsprechende Übergangssignal bei $m / e=39,1$ belegt. 
den ist. Bezüglich der beiden anderen Wirkqualitäten verhalten sich $\mathbf{A}$ und sein Isosteres fast gleich.

Während die früher von uns beschriebenen Sila-benzhydryläther und deren Derivate auf Grund ihres hydrolytischen Abbaus unter in-vitro-Bedingungen (Tyrode-Lösung, $\mathrm{pH} 7,4,37^{\circ} \mathrm{C}$ ) nur eine kurze Wirkdauer besitzen, ist A über eine Meßdauer von 2 Stdn. stabil.

Da bei $\mathbf{B}$ eine pharmakologische Aktivität nicht meßbar ist, obwohl eine solche auf Grund der Isosterie von der Struktur her durchaus zu erwarten wäre, liegt die Vermutung nahe, daß die Verbindung durch hydrolytische Spaltung der $\mathrm{Si}-\mathrm{N}$-Bindung so rasch zerfällt, daß eine Verdrängung des Agonisten von den Rezeptoren nicht in ausreichendem Maße stattfindet.

Unser Dank gilt dem Verband der Chemischen Industrie, Frankfurt am Main, für die Unterstützung mit Sachmitteln und der Bayer-AG, Leverkusen, für die Überlassung von Chlorsilanen. Besonders danken wir Herrn Dr. H.-U. Rossée und Herrn Prof. Dr. F. Meyer für die pharmakologischen Untersuchungen an den neu dargestellten Verbindungen.

\section{Literatur}

1 5. Mitt.: R. Tacke und U. Wannagat, Mh. Chem. 107, 1265 (1976).

2 Mit Auszügen aus der Dissertation R. Tacke, Techn. Univ. Braunschweig 1974.

3 R. Tacke und $U$. Wannagat, Mh. Chem. 106, 1005 (1975).

4 R. Tacke und $U$. Wannagat, Mh. Chem. 107, 111 (1976).

5 R. Tacke und U. Wannagat, Mh. Chem. 107, 439 (1976).

6 Dissertation H.-U. Rossée, Techn. Univ. Braunschweig 1974.

7 R. Tacke und $U$. Wannagat, in Vorbereitung.

8 R. Tacke und $U$. Wannagat, Mh. Chem. 107, 449 (1976).

9 J.W. Ryan, G.K. Menzie und J.L. Speier, J. Amer. Chem. Soc. 82, 3601 (1960).

10 J.F. Hyde und R.C. Delong, J. Amer. Chem. Soc. 63, 1194 (1941).

11 K. A. Andrianov, V.E. Nikitenkov und N.N. Sokolov, Izvest. Akad. Nauk SSSR, Otdel. Khim. Nauk 1960, 1224; Chem. Abstr. 55, 429 (1961).

12 A. D. Petrov, V. F. Mironov und V. G. Glukhovtsev, Izvest. Akad. Nauk SSSR, Otdel. Khim. Nauk 1954, 1123; Chem. Abstr. 49, 7510 (1955).

13 W. H. Daudt und J. F. Hyde, J. Amer. Chem. Soc. 74, 386 (1952).

14 Z. V. Belyalcova, M. G. Pomerantseva und S. A. Golubtsov, Zh. Obshch. Khim. 35, 1048 (1965); Chem. Abstr. 63, 9978 (1965).

Korrespondenz und Sonderdrucke:

Dr. R. Tacke

Institut für Anorganische Chemie

Technische Universität Braunschweig

Pockelsstraße 4

D.3300 Braunschweig

Bundesrepublik Deutschland 